\title{
NEW CRITERIA FOR FREENESS IN ABELIAN GROUPS
}

\author{
BY
}

PAUL HILL( ${ }^{1}$ )

\begin{abstract}
A new criterion is established for an abelian group to be free. The criterion applies to an ascending chain of free subgroups. The result is used to construct groups that are almost free but not free. In particular, we construct examples that show that the class of free abelian groups is not definable in the logical language $L_{\infty K}$ if $\kappa \leq \kappa_{2}$. In doing so, we take advantage of a recent theorem of P. Eklof.
\end{abstract}

1. Introduction and preliminaries. Unless specified otherwise, our notation and terminology agrees with [3] and/or [4]. There are two well-known criteria for the freeness of countable abelian groups, one due to L. Pontryagin and the other a result of E. Specker (see, for example, [3]). Pontryagin's theorem has been generalized in [8] as follows. The generalization applies to groups of arbitrary cardinality.

Theorem 1.1 [8]. If the abelian group $G$ is the union of a countable chain of pure subgroups $G_{n}$, then $G$ is free if $G_{n}$ is free for each $n$.

Several other criteria for the freeness of abelian groups have been established recently. For example, P. Griffith [6] has proved that a torsion-free group is free if it is the extension of a free group by any subgroup of a totally projective $p$-group. More noteworthy at the time it appeared, although simple proofs exist now in [1] and [12], was the result of G. Nöbeling [13] that the bounded functions from any set $S$ to the integers $Z$ form a free abelian group with respect to pointwise addition. Thus, in view of Specker's theorem, if $B$ denotes the subgroup of $\Pi Z$ consisting of the "bounded sequences", then any countable extension of $B$ in $\Pi Z$ is free. Therefore, if $\operatorname{Hom}(G, Z)$ separates the elements of $G$ in a certain way, then $G$ must be free. The final criterion for freeness that we shall mention in the introduction is one established by the present author in [10].

Theorem 1.2 [10]. Let $\mu$ be a limit ordinal of cardinality not exceeding $\boldsymbol{N}_{1}$. Let

Received by the editors October 3, 1972.

AMS (MOS) subject clas sifications (1970). Primary 20K20; Secondary $20 \mathrm{E} 15$.

Key words and phrases. Groups are abelian, free groups, $\boldsymbol{\kappa}_{1}$-freeness, $\boldsymbol{\kappa}_{2}$-freeness, smooth (ascending) chain, criteria for freeness, equivalence in $L_{\infty K^{*}}$

(1) This research was supported, in part, by NSF Grant GP-29025. 


$$
G_{0} \subseteq G_{1} \subseteq \cdots \subseteq G_{\alpha} \subseteq \cdots, \quad a<\mu
$$

be an ascending chain of free subgroups of $G$, indexed by the ordinal $\mu$. If the conditions

(1) $G_{\beta}=\bigcup_{a<\beta} G_{\alpha}$ when $\beta$ is a limit,

(2) $G=\bigcup_{a<\mu} G_{a^{\prime}}$

(3) $\left|G_{a}\right| \leq \boldsymbol{N}_{1}$, are satisfied, then $G$ is free provided that $G_{a_{+1}} / G_{a}$ is $\boldsymbol{K}_{1}$-free for each a.

What might at first seem an unusual consequence of Theorem 1.2 is that it leads to the construction of some interesting groups that are not free. In fact, Theorem 1.2 yields a large class of $\boldsymbol{N}_{2}$-free groups that are not free. In $\$ 4$, we shall see that there is a close connection here with some questions in infinitary logic. P. Eklof has established necessary and sufficient conditions for an abelian group to be $L_{\infty \kappa}$-equivalent to a free abelian group for any infinite cardinal $\kappa$.

The formulas of the language $L_{\infty K}$ are constructed from function and relation symbols by

(i) taking conjunctions over arbitrary sets of formulas,

(ii) applying the negation symbol,

(iii) quantifying over sets of variables of cardinality less than $\kappa$.

It is well known that the class of free abelian groups is not definable in $L_{\infty K}$ when $\kappa=\mathcal{X}_{0}$, and Eklof has used an example of Griffith to prove that the class of free abelian groups is not definable in $L_{\infty K}$ for $\kappa=\kappa_{1}$. One of the main purposes of this paper is to improve again the recent result of Eklof. We shall show that the class of free abelian groups is not definable in $L_{\infty \kappa}$ for $\kappa=\kappa_{2}$. Furthermore, we venture the following.

Conjecture. For no positive integer $n$ is the class of free abelian groups definable in $L_{\infty K}$ with $\kappa=\boldsymbol{\kappa}_{n}$.

As we have indicated, the preceding conjecture will be verified herein only for $n \leq 2$. The procedure used to prove the conjecture, of course, is to construct a nonfree abelian group $G$ that is equivalent in $L_{\infty K}$ to a free abelian group. In producing such a group $G$, a theorem like Theorem 1.2 comes into play. However, as it stands, Theorem 1.2 is not strong enough to yield the desired example. Thus we need to establish further results in the direction of Theorem 1.2. This is accomplished in the next section. We remark that these results are of considerable interest in their own right. Indeed, they are properly interpreted as uniqueness theorems. In $\$ 3$, the main uniqueness theorem (Theorem 2.3) is used to establish an existence theorem. The existence theorem (Theorem 3.1) then applies directly to verify the conjecture concerning $L_{\infty K}$ for $\kappa=\kappa_{2}$ in $\$ 4$. 
2. The uniqueness theorem: a criterion for freeness. For simplicity of terminology, hereafter "group" will mean "abelian group". We shall frequently use the notion of a smooth chain.

Definition 2.1. An ascending chain

$$
G_{0} \subseteq G_{1} \subseteq \cdots \subseteq G_{\alpha} \subseteq \cdots, \quad \alpha<\mu,
$$

of groups, indexed by an ordinal $\mu$, is smootb if $G_{\beta}=\bigcup_{a<\beta} G_{a}$ whenever $\beta$ is a limit ordinal less than $\mu$.

Using the preceding definition, we recast Theorem 1.2 in a new and slightly stronger form.

Proposition 2.1. Let

$$
G_{0} \subseteq G_{1} \subseteq \cdots \subseteq G_{\alpha} \subseteq \cdots, \quad a<\mu,
$$

be a smooth chain of free groups, where $\mu$ has cardinality not exceeding $\boldsymbol{N}_{1}$. If $G_{a+1} / G_{a}$ is $\aleph_{1}$-free of cardinality at most $\kappa_{1}$ for each $a<\mu$, then $G=\bigcup_{a<\mu} G_{a}$ is necessarily free.

Proof. Since $G_{0}$ is free, we can write $G_{0}=\Sigma_{i \in I}\left\langle X_{i}\right\rangle$. The hypothesis implies that $G / G_{0}$ has cardinality at most $\boldsymbol{K}_{1}$. Therefore, there is a subset $J$ of $I$ such that $G=H+K$, where $|K| \leq \aleph_{1}$ and $H=\Sigma_{i \in J}\left\langle X_{i}\right\rangle$. In order to prove that $G$ is free, it suffices to prove that $G$ is $\aleph_{2}$-free. The latter result is a consequence of the following more general proposition.

Proposition 22. Let $G$ be the union of a smooth chain of free groups

$$
G_{0} \subseteq G_{1} \subseteq \cdots \subseteq G_{a} \subseteq \cdots, \quad a<\mu,
$$

where $\mu$ is an arbitrary limit ordinal. If $G_{a_{+1}} / G_{a}$ is $\kappa_{1}-$ free for each $\alpha<\mu$, then $G$ is $\boldsymbol{N}_{2}$-free.

Proof. Let $H$ be a subgroup of $G$ such that $|H| \leq \boldsymbol{\aleph}_{1}$. We need to prove that $H$ is free. There is no loss of generality in assuming that $|\mu| \leq \boldsymbol{N}_{1}$ because $G_{\beta} / G_{a}$ is $\mathcal{N}_{1}$-free whenever $\alpha<\beta<\mu$. Hence we can choose a smooth subchain of length $\aleph_{1}$ that contains $H$. Now we define $H_{\alpha}=H \cap G_{\alpha}$ and apply Theorem 1.2 to the ascending chain

$$
H_{0} \subseteq H_{1} \subseteq H_{2} \subseteq \cdots \subseteq H_{a} \subseteq \cdots, \quad \alpha<\mu,
$$

to conclude that $H=\bigcup_{a<\mu} H_{a}$ is free. 
The existence of a nonfree $\boldsymbol{K}_{2}$-free group has recently been proved independently by Hill [10] and Griffith [7]. In fact, Griffith proved the existence of an $\boldsymbol{x}_{n}$-free group for every positive integer $n$. We want to choose an $\boldsymbol{\kappa}_{2}$-free group that is not free but has a good approximation by free groups. The following provisional definition is employed.

Definition 2.2. A group $G$ is admissible if (1) $G$ is not free and (2) $G$ is the union of a smooth chain

$$
G_{0} \subseteq G_{1} \subseteq \cdots \subseteq G_{a} \subseteq \cdots, \quad a<\omega_{2},
$$

of free subgroups $G_{a}$ such that, for each $\alpha<\omega_{2}$,

(a) $\left|G_{a}\right| \leq \kappa_{1}$, and

(b) $G_{a+1} / G_{a}$ is $\kappa_{1}$-free.

Two important facts about admissible groups are collected in the next theorem.

Theorem 2.1 ([10], [11]). Admissible groups exist and they are $\boldsymbol{\kappa}_{2}-$ free.

Actually, only the existence is proved in [10] and [11], but Proposition 2.2 implies that an admissible group is $\boldsymbol{\kappa}_{2}$-free.

We are now prepared for the following uniqueness theorem.

Theorem 2.2. Let $\mu$ be a limit ordinal of cardinality not exceeding $\boldsymbol{N}_{1}$. If $G$ is the union of a smooth chain

$$
G_{0} \subseteq G_{1} \subseteq \cdots \subseteq G_{a} \subseteq \cdots, \quad a<\mu
$$

of free groups, indexed by $\mu$, such that $G_{a_{+1}} / G_{a}$ is admissible for each $\alpha<\mu$, then $G$ is free.

Proof. We know already from Proposition 2.2 that $G$ is $\boldsymbol{K}_{2}$-free. However, to prove the freeness of $G$, we have to use the hypothesis that the groups $G_{a_{+1}} / G_{a}$ are admissible. For each $a<\mu$, let $G_{a_{+1}} / G_{a}$ be the union of the smooth chain

$$
A_{a, 0} \subseteq A_{a, 1} \subseteq \cdots \subseteq A_{a, \gamma} \subseteq \cdots, \quad \gamma<\omega_{2},
$$

of free subgroups $A_{a, \gamma}$, where $A_{a, \gamma+1} / A_{a, y}$ is $\boldsymbol{\kappa}_{1}$-free and $\left|A_{a, \gamma}\right| \leq \boldsymbol{\kappa}_{1}$.

Let $\mathcal{X}$ denote the collection of subgroups $H$ of $G$ satisfying the following conditions for each $a<\mu$, where $G_{a}=\Sigma_{i \in I(a)}\left\langle X_{i}\right\rangle$.

(i) $H \cap G_{a}=\Sigma_{i \in J(a)}\left\langle X_{i}\right\rangle$ for some subset $J(a)$ of $I(a)$.

(ii) $\left\langle H \cap G_{a+1}, G_{a}\right\rangle / G_{a}=A_{a, y}$ for some $\gamma$ depending on the choice of $H$.

We point out the following crucial property of the collection $X$. If $H \in \mathcal{X}$ and if $A$ is any subgroup of $G$ having cardinality not exceeding $\boldsymbol{K}_{1}$, there exists 
$K \in \mathcal{X}$ such that $K \supseteq\langle H, A\rangle$ and $|K / H| \leq \kappa_{1}$. Without going into details, we explain this as follows. There are at most $\boldsymbol{N}_{1}$ ordinals a with which to deal. Further, in the quotient $G_{a+1} / G_{a}$ any subgroup of cardinality $\kappa_{1}$ and any $A_{a, y}$ can be captured in another $A_{a, \delta}$ since $\left|A_{a, \gamma}\right| \leq \boldsymbol{\kappa}_{1}$ for each $\gamma$. Recall that $\gamma$ ranges over the ordinals of cardinality at most $\kappa_{1}$. Therefore we can use the back-andforth procedure to verify the claim concerning $X$. An essential requirement here obviously is that all the chains involved are smooth. Clearly, the collection $X$ also has the property that it is closed with respect to ascending unions. In view of the properties of $X$ that we have mentioned, in order to show that $G$ is free it remains only to prove that $G / H$ is $\boldsymbol{\aleph}_{2}$-free for each $H \in \mathcal{X}$. This suggests the employment of Proposition 2.2. We need a chain of free groups leading up to $G / H$. Consider

$$
\left\langle G_{0}, H\right\rangle / H \subseteq\left\langle G_{1}, H\right\rangle / H \subseteq \cdots \subseteq\left\langle G_{\alpha}, H\right\rangle / H \subseteq \cdots, \quad a<\mu .
$$

By design, $\left\langle G_{a}, H\right\rangle / H \cong G_{a} / G_{a} \cap H$ is free since $H \in \mathcal{X}$. Furthermore,

$$
\left(\left\langle G_{a+1}, H\right\rangle / H\right) /\left(\left\langle G_{\alpha}, H\right\rangle / H\right) \cong\left(G_{a+1} / G_{a}\right) / A_{a, \gamma},
$$

which is $\boldsymbol{K}_{1}$-free since $A_{a, \gamma+1} / A_{a, \gamma}$ is $\boldsymbol{\aleph}_{1}$-free for each $\gamma$. We conclude, by Proposition 2.2, that $G / H$ is $\boldsymbol{N}_{2}$-free, and $G$ is free.

The next two lemmas enable us to generalize Theorem 2.2 to ordinals $\mu$ that are cofinal with $\omega_{0}$ or $\omega_{1}$ but have cardinality $\kappa_{2}$.

Lemma 2.1. Let $\mu$ be an ordinal of cardinality not exceeding $\boldsymbol{N}_{2}$, and let

$$
A_{0} \subseteq A_{1} \subseteq \cdots \subseteq A_{\alpha} \subseteq \cdots, \quad \alpha<\mu
$$

be a smooth chain of admissible groups. If $A_{a+1} / A_{a}$ is $\kappa_{1}$-free for each $\alpha$, then $B=\bigcup_{a<\mu} A_{a}$ is admissible.

Proof. Since $|\mu| \leq \boldsymbol{N}_{2}$ by hypothesis and since we can replace the original chain by any smooth cofinal subchain without losing the hypothesis, there is no loss of generality in assuming that $\mu \leq \omega_{2}$. First assume $\mu=\omega_{2}$. In view of Theorem 1.2, an immediate consequence of the definition of admissible groups is that they have cardinality exactly $\boldsymbol{\kappa}_{2}$. Hence $B$ has cardinality $\boldsymbol{\kappa}_{2}$ since $A_{\alpha}$ is admissible for each $a$. For $\alpha<\omega_{2}$, let $A_{a}$ be the union of the smooth chain

$$
A_{a, 0} \subseteq A_{a, 1} \subseteq \cdots \subseteq A_{\alpha, \beta} \subseteq \cdots, \quad \beta<\omega_{2},
$$

of free groups with $A_{a, \beta+1} / A_{a, \beta} \boldsymbol{N}_{1}$-free and $\left|A_{a, \beta}\right| \leq \boldsymbol{\kappa}_{1}$.

Since $B$ has cardinality $\mathcal{K}_{2}, B$ is the union of a smooth chain 


$$
B_{0} \subseteq B_{1} \subseteq \cdots \subseteq B_{a} \subseteq \cdots, \quad \alpha<\omega_{2}
$$

of subgroups $B_{a}$ such that, for each $a<\omega_{2}$, the following conditions hold.

(1) $\left|B_{a}\right| \leq K_{1}$.

(2) $B_{a} \subseteq A_{a}$.

(3) If $\alpha$ is isolated, $B_{a}=A_{a, \beta}$ for some $\beta$.

(4) For all $\gamma>a, B_{\gamma} \cap A_{a}=A_{a, \beta}$ for some $\beta$.

(5) For all $\gamma>\alpha,\left\langle B_{\gamma}, A_{a}\right\rangle$ is pure in $B$.

The main point in the development of the prescribed chain is that conditions (4) and (5) are required only for $\boldsymbol{N}_{1}$ different ordinals $a$ when $\gamma$ is fixed. We remark that $A_{a}$ is necessarily pure in $B$, and refer to [9] for the purification (5).

Note that condition (3) implies that $B_{a}$ is free since $A_{a, \beta}$ is free. To show that $B_{a+1} / B_{a}$ is $X_{1}$-free, it suffices to prove that both $B_{a+1} / A_{a} \cap B_{a+1}$ and $A_{a} \cap B_{a+1}^{-} / B_{a}$ are $\aleph_{1}$-free since an extension of an $\boldsymbol{X}_{1}$-free group by an $\boldsymbol{X}_{1}$-free group is again $\boldsymbol{K}_{1}$-free. The relations

$$
B_{a+1} / A_{a} \cap B_{a+1} \cong\left\langle A_{a}, B_{a+1}\right\rangle / A_{a} \subseteq A_{a+1} / A_{a}
$$

show that $B_{a+1} / A_{a} \cap B_{a+1}$ is $\boldsymbol{K}_{1}$-free. Furthermore, if $\alpha$ is isolated, then $A_{\alpha} / B_{a}=A_{d} / A_{a, \beta}$ is $\boldsymbol{\kappa}_{1}$-free. If $\alpha$ is a limit, then $A_{\alpha} / B_{a}$ is the union of the smooth chain

$$
\left\langle A_{0}, B_{\alpha}\right\rangle / B_{\alpha} \subseteq\left\langle A_{1}, B_{\alpha}\right\rangle / B_{\alpha} \subseteq \cdots \subseteq\left\langle A_{\lambda}, B_{\alpha}\right\rangle / B_{\alpha} \subseteq \cdots, \quad \lambda<a .
$$

Since $\left\langle A_{\lambda}, B_{a}\right\rangle / B_{a} \simeq A_{\lambda} / A_{\lambda, \delta}$ is $\boldsymbol{K}_{1}$-free and since $\left\langle A_{\lambda}, B_{a}\right\rangle$ is pure, it follows that $A_{\alpha} / B_{a}$ is $\boldsymbol{\kappa}_{1}$-free. Thus, in any case, $\left(A_{a} \cap B_{a+1}\right) / B_{a}$ is $\boldsymbol{\kappa}_{1}$-free, and so is $B_{a+1} / B_{a}$. We have shown that $B$ meets the requirements for an admissible group with it being obvious that $B$ cannot be free since it contains an admissible subgroup.

If $\mu<\omega_{2}$, the argument is modified by requiring of the groups $B_{a}$ only condition (1) and conditions (4) and (5) for all $y<\mu$. Again, it can be shown that $B_{a}$ is free and $B_{a+1} / B_{a}$ is $\boldsymbol{K}_{1}$-free.

The proof of the next lemma is omitted.

Lemma 2.2. An extension of an admissible group by an admissible group is admissible.

Now we present the main uniqueness theorem, which is required in the sequel.

Theorem 2.3. Let $\mu$ be a limit ordinal of cardinality not exceeding $\boldsymbol{N}_{2}$ that is cofinal with $\omega_{0}$ or $\omega_{1}$. If $G$ is the union of a smooth chain 


$$
G_{0} \subseteq G_{1} \subseteq \cdots \subseteq G_{a} \subseteq \cdots, \quad \alpha<\mu
$$

of free groups such that $G_{a_{+}} / G_{\alpha}$ is admissible for each $a$, then $G$ is free.

Proof. Choose a smooth cofinal subchain

$$
H_{0} \subseteq H_{1} \subseteq \cdots \subseteq H_{a} \subseteq \cdots, \quad \alpha<\omega_{i}, i=0 \text { or } 1 .
$$

Then $H_{a+1} / H_{a}=G_{\delta} / G_{\gamma}$ for appropriate $\gamma$ and $\delta<\mu$. By Lemma 2.1 and Lemma $2.2, G_{\delta} / G_{\gamma}$ is admissible, by induction on $\delta$, since $G_{\delta} / G_{\gamma}$ is the union of the chain

$$
G_{\gamma+1} / G_{\gamma} \subseteq G_{\gamma+2} / G_{\gamma} \subseteq \cdots \subseteq G_{\gamma+\alpha} / G_{\gamma}, \quad \gamma+a<\delta
$$

of admissible groups when $\delta$ is a limit, and $G_{\delta} / G_{\gamma}$ is the extension of $G_{\delta-1} / G_{\gamma}$ by $G_{\delta} / G_{\delta_{-1}}$ otherwise. We can now apply Theorem 2.2 to conclude that $G=$ $\bigcup_{H_{a}}$ is free.

3. The existence theorem. To remove skepticism early about admissible groups, we have already presented the existence theorem (Theorem 2.1) for admissible groups, which is an essential ingredient for the following result.

Theorem 3.1. There exist torsion-free groups $G$ satisfying the following conditions.

(I) $G$ is not free.

(II) If $H$ is a subgroup of $G$ baving cardinality not exceeding $\kappa_{1}$, then $H$ is contained in a free subgroup $F$ of $G$ such that $G / F$ is $N_{2}$-free.

Proof. Although we have not mentioned this before, it should be evident that Theorem 2.3 cannot hold for all ordinals $\mu$. Indeed, suppose the theorem is valid for all ordinals $\mu$ of cardinality not exceeding $\kappa_{2}$. Using the existence theorem for admissible groups, we can construct a smooth chain

$$
G_{0} \subseteq G_{1} \subseteq \cdots \subseteq G_{\alpha} \subseteq \cdots, \quad \alpha<\omega_{3}
$$

of free groups $G_{\alpha}$ of cardinality $\boldsymbol{N}_{2}$ so that $G_{\alpha+1} / G_{\alpha}$ is admissible. There is no difficulty with this construction since freeness is preserved at limit ordinals under the assumption of the validity of the generalization of Theorem 2.3 to all ordinals $\mu<\omega_{3}$. It now becomes obvious that Theorem 2.3 must fail for $\mu=\omega_{3}$ since $G=\cup_{a<\omega_{3}} G_{a}$ cannot be free. If $G$ were free, then $G_{a}$ would have to be a direct summand of $G$ for some $a<\omega_{3}$. This implies that $G / G_{a}$ is free and that the subgroup $G_{a+1} / G_{a}$ is also free. The latter contradicts the choice of $G_{a+1} / G_{a}$ as an 
admissible group. We conclude: Theorem 2.3 fails for some ordinal $\mu \leq \omega_{3}$.

Let $\mu \leq \omega_{3}$ be the first ordinal for which Theorem 2.3 fails. With the same notation of Theorem 2.3, $G=\bigcup_{\alpha<\mu} G_{\alpha}$ is not free by the choice of $\mu$. An essential point is that $\mu$ is not cofinal with $\omega_{0}$ nor $\omega_{1}$. We claim that $G$ satisfies condition (II) of Theorem 3.1. Let $H$ be a subgroup of $G$ having cardinality $\boldsymbol{N}_{1}$. Since $\mu$ is not cofinal with $\omega_{0}$ nor $\omega_{1}, H$ is contained in $G_{a}$ for some $\alpha<\mu$. To complete the proof of Theorem 3.1, it suffices to prove that $G / G_{a}$ is $\aleph_{2}$-free for $\alpha<\mu$. Let $A$ be a subgroup of $G / G_{\alpha}$, where $|A| \leq \aleph_{1}$. If $a<\lambda<\mu$, define $A_{\lambda}=$ $\left(G_{\lambda} / G_{a}\right) \cap A$. Recall from Theorem 2.1 that admissible groups are $\boldsymbol{K}_{2}$-free. Since $A_{a+1}$ is isomorphic to a subgroup of $G_{a+1} / G_{a}, A_{a+1}$ must be free. Likewise, $A_{\lambda+1} / A_{\lambda}$ is isomorphic to a subgroup of $G_{\lambda+{ }_{1}} / G_{\lambda}$ and, therefore, is free. The smoothness of the chain of groups $A_{\lambda}$ implies that $A=\bigcup_{\lambda \leq \mu} A_{\lambda}$ is free. Thus $G / G_{a}$ is $\boldsymbol{X}_{2}$-free, and Theorem 3.1 is proved.

4. Freeness in $L_{\infty K^{*}}$ As we indicated earlier, Eklof has established necessary and sufficient conditions for a group to be equivalent in $L_{\infty K}$ to a free group, and we are indebted to him for a preprint of [2].

Theorem 4.1 [2]. For any infinite cardinal $\kappa$, an abelian group $G$ is $L_{\infty K^{-}}$ equivalent to a free abelian group if and only if: Every subgroup $H$ of $G$ generated by less than $\kappa$ elements is contained in a free subgroup $F$ of $G$ with the property that $F$ splits out of $K$ whenever $F \subseteq K \subseteq G$ and $K / F$ is generated by less than $\kappa$ elements.

The preceding theorem and an example of Griffith [5] imply that the class of free abelian groups is not definable in $L_{\infty} \boldsymbol{N}_{1}$. We offer the improvement promised earlier.

Theorem 4.2. The class of free abelian groups is not definable in $L_{\infty K}$ for $\kappa=\boldsymbol{N}_{2}$.

Proof. Let $G$ be any group satisfying conditions (I) and (II) of Theorem 3.1. Then $G$ is not free, but $G$ is equivalent to a free group in $L_{\infty} \boldsymbol{N}_{2}$ because the $\boldsymbol{N}_{2}$-freeness of $G / F$ immediately implies the splitting condition of Theorem 4.1 when $\kappa=\boldsymbol{x}_{2}$.

We can strengthen further the preceding theorem in the following manner.

Theorem 4.3. There exist abelian groups that are not free, but have the property that every subgroup is equivalent in $L_{\infty N_{2}}$ to a free abelian group.

Proof. We simply need to analyze closer the groups $G$ that are associated with the failure of Theorem 2.3 at the smallest possible ordinal $\mu$. Recall that $\mu$ 
is either cofinal with $\omega_{2}$ or else $\mu=\omega_{3}$. Thus if $G^{\prime}$ is a subgroup of $G$ and if $H^{\prime}$ is a subgroup of $G^{\prime}$ having cardinality $\aleph_{1}$, then $H^{\prime} \subseteq G^{\prime} \cap G_{a}$ for some $a<$ $\mu$. Since $G^{\prime} \cap G_{\alpha}$ is free and $G^{\prime} / G^{\prime} \cap G_{a} \cong\left\langle G^{\prime}, G_{\alpha}\right\rangle / G_{a}$ is $\aleph_{2}$-free, the rest follows.

\section{REFERENCES}

1. G. Bergman, Boolean rings of projection maps, Proc. London Math. Soc. 4 (1972), 593-598.

2. P. Eklof, Infinitary equivalence of abelian groups: With counterexamples to Scott's theorem in uncountable cardinals (preprint).

3. L. Fuchs, Infinite abelian groups. Vol. I, Pure and Appl. Math., vol. 36, Academic Press, New York, 1970. MR 41 \#333.

4. P. Griffith, Infinite abelian group theory, University of Chicago Press, Chicago III. and London, 1970. MR $44 \# 6826$.

5. - A note on a theorem of Hill, Pacific J. Math. 29 (1969), 279-284. MR 39 \#6919.

6. - Extensions of free groups by torsion groups, Proc. Amer. Math. Soc. 24 (1970), 677-679. MR 41 \#1879.

7. - $\boldsymbol{K}_{n}$-free abelian groups (preprint).

8. P. Hill, On the freeness of abelian groups: A generalization of Pontryagin's theorem, Bull. Amer. Math. Soc. 76 (1970), 1118-1120. MR 41 \#8518.

9. - The purification of subgroups of abelian groups, Duke Math. J. 37 (1970), 523-527. MR $42 \# 365$.

10. - The splitting of modules and abelian groups, Canad. J. Math. (to appear).

11. - Sums of cyclic groups, Lecture Notes, University of Arizona, Tucson, Ariz., April 1972.

12. - The additive group of commutative rings generated by idempotents, Proc. Amer. Math. Soc. 38 (1973), 499-502.

13. G. Nöbeling, Verallgemeinerung eines Satzes von Herrn E. Specker, Invent. Math. 6 (1968), 41-55. MR 38 \#233.

DEPARTMENT OF MATHEMATICS, FLORIDA STATE UNIVERSITY, TALLAHASSEE, FLORIDA 32306 